\title{
USING ASYNCHRONOUS AUDIO FEEDBACK TO ENHANCE TEACHING PRESENCE AND STUDENTS' SENSE OF COMMUNITY
}

\author{
Philip Ice \\ Department of Middle, Secondary and K-12 Education \\ College of Education, University of North Carolina Charlotte
}

\section{Reagan Curtis}

Department of Technology, Learning, and Culture

College of Human Resources and Education, West Virginia University

\author{
Perry Phillips \\ Department of Curriculum \& Instruction / Literacy Studies \\ College of Human Resources and Education, West Virginia University
}

\author{
John Wells \\ Department of Teaching and Learning \\ School of Education, Virginia Polytechnic Institute and State University (Virginia Tech)
}

\begin{abstract}
This paper reports the findings of a case study in which audio feedback replaced text-based feedback in asynchronous courses. Previous research has demonstrated that participants in online courses can build effective learning communities through text based communication alone. Similarly, it has been demonstrated that instructors for online courses can adequately project immediacy behaviors using textbased communication. However, we believed that the inclusion of an auditory element might strengthen both the sense of community and the instructor's ability to affect more personalized communication with students. Over the course of one semester, students in this study received a mixture of asynchronous audio and text-based feedback. Our findings revealed extremely high student satisfaction with embedded asynchronous audio feedback as compared to asynchronous text only feedback. Four themes, which accounted for this preference, were culled out in an iterative, inductive analysis of interview data: 1. Audio feedback was perceived to be more effective than text-based feedback for conveying nuance; 2 . Audio feedback was associated with feelings of increased involvement and enhanced learning community interactions; 3. Audio feedback was associated with increased retention of content; and 4. Audio feedback was associated with the perception that the instructor cared more about the student. Document analysis revealed that students were three times more likely to apply content for which audio commenting was provided in class projects than was the case for content for which text based commenting was provided. Audio commenting was also found to significantly increase the level at which students applied such content. Implications of this case study and directions for future research are addressed in the discussion and conclusions section of this paper.
\end{abstract}




\section{KEYWORDS}

Online Learning, Personalized Communication, Student Satisfaction, Embedded Asynchronous Audio Feedback, Nuance, Retention of Content, Instructor Caring

\section{INTRODUCTION}

As the number of online courses continues to expand, so must the ways in which instructors engage in active facilitation of learning among their students. This study focuses on one aspect of facilitation, the way in which we communicate and guide students in asynchronous learning networks (ALN) and how this process might be improved upon.

While the evolution of ALN has made it increasingly easier to involve remotely based students in twoway communications [1] and enable students to process more complex information [2], instructors are often required to adapt to new roles [3]. While several frameworks have been developed to explain the role of the instructor [3, 4, 5], a system first proposed by Berge [6] and later refined by others [7] proposes a four part model consisting of pedagogical, social, technical, and managerial dimensions, each with a varying number of roles. For purposes of this study, the social dimension and three roles (profession-inspirer, feedback-giver, and interaction-facilitator) within the pedagogical dimension are considered the most important. These are depicted in the following table which was derived from work by Liu and colleagues [2].

\begin{tabular}{|l|l|l|}
\hline Dimensions & Roles & Description of Roles \\
\hline Pedagogical & Profession-inspirer & $\begin{array}{l}\text { Promote professional dialogue among online learners; relate } \\
\text { personal experiences and cases to the discipline; point to } \\
\text { professional organizations. }\end{array}$ \\
\hline & Feedback-giver & $\begin{array}{l}\text { Provide timely and high quality feedback; provide } \\
\text { formative feedback for continuous learning engagement. }\end{array}$ \\
\hline Social & Social rapport builder & $\begin{array}{l}\text { Facilitate peer interaction in online discussion through a } \\
\text { wide range of facilitation strategies. } \\
\text { learning community. }\end{array}$ \\
\hline
\end{tabular}

Table 1. Select Roles of Online Instructors

In the traditional face-to-face classroom setting, each of these roles would be dependent upon both verbal and non-verbal cues. In the online environment, however, the primary form of communication is via text and therefore devoid of traditional paralinguistic cues [2]. Arbaugh [8] suggests that the relative low richness of text-based communication may make interdependent, ambiguous tasks particularly challenging.

Critics of online learning, building on the low richness of text-based communication, contend that because interactions occur in a disembodied form, this lack of nuance leads to a loss of meaning [9, 10, $11,12]$. As such, it is argued that asynchronous learning is not sufficiently rich in the socially mediated practice that Vygotsky [13] described as necessary to construct knowledge. However, this narrow interpretation of Vygotsky discounts the ability of learners to conceptualize "being" as anything other than a physical construct. 
The ability to project oneself through various media, termed social presence, was initially described by Short, Williams and Christie [14] who proposed that, as critics of asynchronous learning contend, the ability to project verbal and nonverbal information directly impacted the degree to which presence was perceived. However, Rourke, Anderson, Garrison and Archer [15] and Swan [16] argued that this may not be the case as learners in online courses appeared to build effective learning communities by projecting their personalities through text alone.

Lombard and Dutton [17] viewed this creation of a presence in online courses as the ability to project oneself into a virtual. In an extension of this concept, Laffey, Lin and Lin [18] described the social element of asynchronous communication evolving as learners come to view their interactions with tasks and tools as being a fluid, integrated process rather than as a series of tasks. They compared this process to a speaker interacting with others in a foreign language. The more fluent the speaker becomes with the new language the less difficult interactions become. Theoretically, this would mean that the technologies become part of the interaction itself and are therefore not viewed as objects upon which learners have to act to create virtual embodiments [19].

Gunawardena and Zittle [20] found that the sense of "being there" was established in the online environment through providing and interpreting emoticons as a replacement for nuance and nonverbal cues. Using a 14-item questionnaire, they found $60 \%$ of the variance in student satisfaction was attributable to perceptions of social comfort and presence. Rovai [21] explained that this type of satisfaction can occur when text based, socio-emotional-driven interactions promote a sense of connectedness among learners in asynchronous learning networks (ALN).

Richardson and Swan [22] used regression analyses to determine the relationship between perceived social presence and perceived learning. Analysis of data collected from 17 courses revealed that $46 \%$ of the variability in perceived learning could be predicted by student perceptions of social presence. However, the study also revealed that an even stronger relation $\left(\mathrm{R}^{2}=0.53\right)$ existed between perceived learning and overall satisfaction with the instructor. This finding indicated that satisfaction with the instructor was at least as important as was perceived social presence. Further, the authors found that a strong relation $\left(R^{2}=0.36\right)$ existed between students' perceptions of social presence and satisfaction with the instructor. Based on these findings it was concluded that 'students' perceptions of social presence were related to the perceptions of their instructors as having a satisfactory online presence in terms of amount of interaction and/or quality of that interaction.”

Through factor analysis, Arbaugh [23] found instructor immediacy behaviors in online courses were a significant predictor of student learning. Based on Gorham's [24] verbal immediacy scale, Arbaugh defined immediacy behaviors as being comprised of two parts. The first, classroom demeanor, "reflected the instructor's use of personal examples, humor, and openness toward and encouragement of student ideas and discussion." The second, name recognition, referred to the "extent to which the instructor was addressed by name by the students and vice versa.”

\section{A. Instructional Design Features that Foster Community}

Informed by the studies previously discussed, we have been improving on our design of ALN instruction to facilitate meaningful discourse and create dynamic learning environments. Specifically, in our courses over the past six semesters, we have attempted to incorporate recommendations found in the literature related to the projection of teaching presence through immediacy behaviors. Surveys of student satisfaction from these courses indicated that students were generally highly satisfied with our efforts and students' qualitative feedback, when provided, typically made us believe we were doing a good job of 
creating a rich learning environment. However, even if social presence is strong, student may prefer even more interactive communication [25, 26], and we question whether greater interaction might also apply to the projection of teaching presence. Arbaugh [8] found media variety to be positively associated with perceived learning among students in web-based MBA courses. In a review of the literature, Liaw and Haung [27] suggested that presentation of web-based course content through a variety of media positively impacted learner experiences.

Ideally, we would have liked to have used an asynchronous videoconferencing mechanism similar to that envisioned by Watt, Walther and Nowak [28]. Extending work by Walther and Burgoon [29], Watt and colleagues wrote that such a system would take full advantage of both verbal and nonverbal cues thereby increasing copresence; "the sense that one is actively being perceived and that one is actively perceiving another [28]." However, based on previous student surveys we knew that approximately one third of our students were likely to be taking classes via dialup connections, making the use of streaming video impractical. Thus, the only feasible alternative available to us was the use of asynchronous audio.

Research on the use of stand alone audio in ALN, especially audio feedback, is rather limited. The study that provided us with the most insight as to how audio feedback might be perceived by students was conducted by Jelfs and Whitelock [30]. These researchers created a virtual environment in which various navigational techniques were used. All of the participants indicated in follow-up interviews that the preprogrammed auditory feedback was as important to their success and satisfaction with the environment as was ease of navigation. Significantly, these two factors were considered to be even more important than interactivity or previous experience.

\section{B. Use of Audio Feedback}

Use of audio commenting in the face-to-face classroom can be traced to at least 1982, when Olson [31] reported using the technique in English courses at a two year college. In a discussion of the technique, Olson opined that his students believed audio commenting reflected a sense of caring on the part of the instructor that extended beyond their written products. The ability to project through tone of voice, he argued, enabled the instructor "to be more supportive and caring."

Building on Olson's work, Mellen and Summers [32] provided students in an English course with tapes containing audio feedback and conducted surveys and interviews at the end of the semester. Results demonstrated that students were likely to view audio feedback as being positive regardless of the context. Additionally, $70 \%$ of students reported that they felt encouraged to revise their work as a result of receiving auditory feedback and 54\% felt more confident about their writing. These findings provide strong, highly positive indicators of student perceptions regarding the use of audio feedback and point to its potential as a tool in asynchronous online courses.

In a study of student-student audio based interactions in ALN, Kim [33] found that students had generally positive perceptions of the medium, but that its use decreased motivation. However, audio did increase social presence, a finding that supported earlier research in which Reeves and Nass [34] concluded that human voice increased social presence. In a seeming contradiction, Bargeron and colleagues [34] found that students preferred to use text rather than audio in threaded discussions because they found it easier and quicker to read text messages than listen to audio.

However, the sample size in the study conducted by Bargeron and colleagues [35] was small with only 4 of the 6 total participants indicating a preference for text based feedback. We conducted a pilot study 
asking 83 of our students to complete a survey regarding the relative time required to utilize text based versus audio feedback. We found that 28 students believed it took longer to listen to audio feedback than to read text-based feedback, 35 believed the time required was approximately the same, and 20 believed it took less time to listen to audio feedback. In addition, after answering questions about the time required to listen to audio feedback, 6 students emailed the instructor wishing to clarify their answers. The following is representative of the emails received:

I just finished answering some questions about the time it took to listen to comments or read comments. My answer was that it took longer. However, I wanted to clarify that a little. It took longer because I replayed the comments a couple of times so I could really see what was being said as it related to my work and get more out of it. I don't do this when the comments are written because I don't think they are as good.

Based on these findings, we concluded that the difference in time required to listen to audio feedback versus reading text-based feedback was not a significant factor in deciding whether the technique should be used. In fact, based on the supplemental feedback, there was reason to believe that even though some students perceived audio feedback to be more time consuming, they still preferred it because they believed they got more out of it. Clearly, more research is needed in this area to explore students' perceptions related to each type of feedback.

The research clearly shows connections between perceived learning, perceptions of social presence, instructor satisfaction, and immediacy behaviors in building a sense of community among ALN learners. Yet to be established, however, is the extent to which auditory feedback might further enhance teaching presence and therefore build a stronger student sense of community.

\section{METHOD}

From spring 2004 through summer 2005, we served as instructors in seven asynchronous online courses. Despite being highly satisfied with the experiences and believing that our students had significant learning experiences, we wondered if we had done all we could to make our relationships with students as personal as possible given the constraints of the medium. While we disagree with those who view online learning as detached and impersonal [36, 37], we were concerned about our ability to adequately convey nuance in a manner similar to that which occurs in face-to-face classrooms. This concern prompted our research to better understand the nature of audio feedback in an asynchronous learning network. Specifically, in this study we sought to answer the following set of research questions (RQ):

- RQ 1: Between audio and text-based student feedback in ALN, which do students believe is a more effective means of interaction with their instructor?

- RQ 2: To what degree do students believe audio feedback is an effective replacement of instructor/student interaction that typically occurs in traditional face-to-face classes?

- RQ 3: How does the use of audio feedback impact the sense of community in ALN?

- RQ 4: In what manner is perceived learning impacted by the use of audio feedback?

- RQ 5: What relationship exists between the use of audio feedback and student satisfaction?

\section{A. Instructional Setting}

Curriculum and Instruction 687, Advanced Teaching Strategies, was the course through which this study was conducted. Prior to this study, C\&I 687 had been offered completely online for three consecutive semesters. 
Structurally, C\&I 687 consisted of ten learning units in which students explored and evaluated advanced teaching concepts and strategies. In the first unit, students were introduced to the philosophical foundations of constructivist teaching and asked to evaluate a series of readings with respect to their personal experiences in the classroom. In seven of the remaining units, students were introduced to eight teaching strategies (concept attainment, inductive learning, cooperative learning, synectics, direct instruction, mnemonics and classroom discussion) through readings that addressed methodology, through text and video based case study analysis and through discussion postings in which students were asked to apply the various models to content area lesson plans of their choice. Students then evaluated each other's postings and refined lesson plan strategies based on the communal knowledge constructs that emerged.

One of the two remaining units was a mid-term assessment activity where students selected two videobased classroom vignettes and conducted an evaluative case study for which they identified the teaching strategies employed, explained the usage rationale and suggested how the teacher might have improved the manner in which their students acquired knowledge. The final unit consisted of two parts: part 1 consisted of six reflective activities in which students were asked to evaluate how praxis might be impacted by contemporary and emerging societal and technical issues; the second part of the final unit required groups of students to develop a series of thematic, interdisciplinary lesson plans in which strategies explored during the semester were utilized. These plans required that students use a minimum of three teaching strategies explored during the semester. After all projects were submitted, students were expected to evaluate plans submitted by other groups and suggest revisions.

The course was a major elective for both master's and doctoral level students in the Curriculum and Instruction program. The course had no prerequisites and was taken at various times during students' plan of study.

In previous years when this course was taught, feedback was provided to students in two ways. In the first, the instructor would interact with the students' text based postings on the discussion board using Socratic questioning to enhance and expand upon various threads that emerged. Additional group feedback was provided at the conclusion of each thread. In the second, the instructor would provide individualized text based feedback via email to students on each discussion topic or submission.

\section{B. Use of Audio Commenting Within the Instructional Setting}

In addition to utilizing approaches to text-based feedback from previous years, we incorporated audio commenting in this iteration of the course. When posting audio comments to the discussion board, in emails to the entire class, or to small groups, the instructors produced wav files using Audacity freeware. The files were then added to the discussion board or email as attachments.

In the case of individualized feedback, the instructors selected various discussion posts made by a student, copied them to a Word document, inserted comments and sent the document back to the student via course email. This type of individualized commenting was also used for the midterm case studies, final reflections and the group project.

We provided approximately half of the individualized feedback in a text-based format and the other half via audio. At the end of the course all students had received six documents in which text feedback was used and five in which audio feedback was used. To avoid the introduction of bias, prior to the beginning of the semester each assignment was given a number from one to 12. These numbers were then entered into excel and randomized. From this list, we assigned alternating text-based or audio feedback as the 
modality that would be used.

On the discussion board, we engaged in Socratic questioning as in previous semesters. At the end of the semester, the discussion board contained a total of 1471 postings and replies. Of these, 203 were Socratictype questions that we posed to students on an individual basis. In addition, we provided another 59 postings that took the form of group feedback: 31 of these were text-based and 28 used audio.

Technically, the audio feedback was produced by first copying select discussion board postings into a Word document or opening a Word document in which students had submitted individual assignments. The Word document was then converted into a PDF document using Adobe Acrobat Pro 7. Once in this format, the instructor used the Record Audio Comment tool within the Comment and Markup option. Depending on a host of factors, including length, number of topics discussed and quality of the work submitted, the instructor placed varying numbers of audio files within the document, as well as a summary statement at the end of each document. The audio feedback was spontaneous in nature, as it was intended to replicate the non-scripted verbal interactions that occur in F2F environments.

In the instances where students received text feedback, it was in the form of a PDF document using the Note Tool selected from the Comment and Markup option. Text comments were placed at various points throughout the document and at the end, in a fashion mirroring that used in the audio feedback. The same document format and comment placement strategies were used to ensure that any difference in perceptions of the commenting modality would not be influenced by these extraneous variables.

To determine what impact using audio commenting had on time required to provide feedback, we maintained a log of the amount of time required to provide both text-based and audio feedback. During the analysis of data, we also compared the volume of audio and text based feedback that was provided to students.

\section{Participants}

West Virginia University's Institutional Review Board approved the protocol for this study to ensure ethical treatment of all participants. For the semester in which this study occurred, enrollment consisted of 26 master's level students and 8 doctoral students. Of the 26 master's students, 17 were practicing teachers and 9 pre-service teachers. Geographically, 29 of the students who took the course were located in West Virginia, 3 were located in Maryland, 1 in Alabama and 1 was on military deployment in Djibouti.

An email was sent to all students during the last week of the course asking for volunteers to participate in post-course interviews. Seven doctoral students, 15 master's level practicing teachers and 5 master's level pre-service teachers volunteered to participate.

\section{Design}

A nested mixed methods design with both concurrent and sequential components was implemented [38]. We gave priority to the qualitative components nesting quantitative data within them in order to enrich our description of participants' perceptions related to audio feedback [39]. Three separate sets of data were originally planned for triangulation during data analysis and interpretation: end of course survey data, post-course interview data and final projects. Unsolicited qualitative feedback generated throughout the semester, though not originally part of the research design, was added as a data set because it 
contained rich and compelling data that could not be ignored. The end of course survey data included both qualitative and quantitative components collected concurrently with the final project data. Interview data gathered sequentially allowed us to follow up on themes generated from the end of course survey results.

We selected a mixed methods research design for our work, and being guided by a "pragmatic approach" or paradigm [40] we sought to capitalize on the strengths of both quantitative and qualitative approaches to data collection. This clearly required following established criteria for generating high quality quantitative and qualitative data. While criteria for judging the quality of quantitative studies are well established, there is less agreement regarding what quality criteria are applicable to qualitative research [41, 42]. Searle [43] argued that triangulation of data sources aimed at enriching understanding through and of multiple perspectives should be the central criteria by which qualitative research is judged. Taking his point, we included multiple forms of qualitative data (survey, interview, and document), blended with quantitative (survey) and quantified (document) data, and analyzed these using strategies designed to achieve triangulation.

\section{Unsolicited Feedback}

During the semester, 14 students sent a total of 16 unsolicited emails to the instructor related to the use of audio feedback. The rich data in these emails provided early insight into how students perceived the modality, as well as technical difficulties that a small number of students were experiencing. The emails were coded and categorized based on thematic similarities that emerged in cross case analyses. Although this was not data originally designed into the study, this unanticipated feedback clearly added to our understanding of students' perceptions of audio feedback. Capitalizing on the emergent nature of qualitative inquiry, this data set was included as an extra point of validation in the triangulation process.

\section{End of Course Survey Data}

At the end of the course, students were asked to complete a survey to assess satisfaction and perceived learning. The survey consisted of 52 items. The first 50, derived from instruments previously developed by Spencer and Thompson [44, 45], addressed student satisfaction with course design, perceived learning and sense of community. Two additional items related specifically to the use of audio feedback were added: 1) a Likert-type scale item addressing student perceptions of the relative effectiveness of audio versus text-based feedback, and 2) an open-ended item soliciting additional comments relative to audio feedback. The Likert-type item was analyzed using descriptive statistics. Responses to the open ended item were coded and thematically categorized using cross case analysis. This analysis then informed the semi-structured post course interview protocols.

To guard against a novelty effect, as is often seen in student satisfaction with online courses [15, 46], we continued to collect data from other courses in which the instructors used audio feedback. This quantitative data consisted of responses to two questions. In the first, "I prefer audio feedback to textbased feedback," students were asked to respond on a five point Likert-type scale with choices ranging from Strongly Disagree to Strongly Agree. The second question asked students how many courses they had previously taken in which audio commenting was used $(0,1,2,3$, or more than 3$)$.

\section{Post Course Semi-structured Individual Interviews}

Of the 34 students enrolled in the course, 27 volunteered to participate in post course interviews. These semi-structured interviews were conducted during the two weeks following the end of the semester. During interviews, individual students were asked their impression of both the course and each type of feedback using an interview protocol guide (see Appendix A) developed following principles described 
by Berg [47] and Patton [48]. Two interviewers were involved in the process to ensure consistency. Indepth probing of responses was conducted on an individualized basis to draw out more detailed data related to why students perceived audio feedback to be more or less effective, as well as how it may have altered their perceptions of what it meant to be a participant in an asynchronous learning network. Interviews lasted approximately 50 minutes and were audio taped using a portable mp3 recorder. After all interviews were complete, transcriptions were generated for coding. The transcribed interview texts were analyzed following suggestions by both Strauss [49] and Tesch [50] using an interpretive, iterative approach with emphasis placed on drawing out thematic strands. Because of the data richness, both within and cross case analyses were utilized to more fully represent what occurred at both the individual level and as part of a group dynamic.

To guard against a novelty effect, check for consistency in themes, and detect new themes, a total of 51 students were randomly selected from 17 courses in which the instructor had used audio commenting since the completion of the original study. These students were emailed a questionnaire (Appendix C) in which they were asked to reply to a series of open ended questions. The questionnaire was derived from the interview protocol used to conduct the post-course interviews (Appendix A). Using an iterative, interpretive process, themes were drawn out in the same manner used for transcribing the original interviews.

\section{Final Project Document Analysis}

The final project for this course required groups of students to develop a series of thematic, interdisciplinary lesson plans that utilized a minimum of three strategies explored during the semester. Document analysis of final projects was conducted by first coding for the types of strategies students chose to use for lesson plan design and then categorizing based on the type of instructor feedback (text versus audio) used when students studied these strategies earlier in the course. The incidences of the various categories were quantified and descriptive statistics calculated to explore how feedback modality might have impacted content usage.

The final projects were then recoded to determine the level of Bloom's taxonomy [51] applied to each strategy. In this process, the lesson plans students developed were decompressed and individual activities evaluated using a rubric derived from Slavin's [52] application of Bloom's taxonomy to pedagogy (see Appendix B). Coded documents were reviewed by two researchers to ensure consistency. The reviewers unanimously agreed on the coding. The results were presented using descriptive statistics to determine if audio feedback impacted the level at which content was used.

\section{Triangulation}

After analyzing each data set in the manner described above, open coding was used to isolate prevalent themes followed by negative case analysis to explore consistency across data sources [53]. First, the results of the quantitative end of course survey question were compared with the findings from the post course interviews and unsolicited feedback for additional confirmation. Next, the findings from analyzing the qualitative question in the end of course survey were crosschecked with the interview data and unsolicited feedback. The end of course survey did not address content retention and so could not be crosschecked with the document analysis. Usage frequency and level counts derived from document analysis were checked for consistency with interview data focused on content retention. The interpretive conclusions from triangulation analyses were then compared to what is known about corresponding elements in learning theory and social presence literature to develop grounded theory that could be applied to future research. 


\section{RESULTS OF THE STUDY}

This study was originally designed with three data sources: end of course survey data, semi-structured interviews and document analysis. However, data rich material in the form of unsolicited feedback from students was included as we found it to lend significant insight into what students thought at the time they were actually receiving the audio feedback. In addition, these unsolicited emails allowed us to refine some of the potential probing areas in the interview guide. Results of analyzing each data source separately are provided below. Triangulation, observations and conclusions follow in section IV.

\section{A. Unsolicited Feedback}

Fourteen students in the course sent a total of 16 unsolicited emails regarding the use of audio feedback. In 14 of these, 11 of which were sent within three days of the initial use of audio feedback, students wrote to express a high degree of satisfaction with the modality. The remaining two emails were related to technical problems with getting the audio files to play. No unsolicited emails expressing negative sentiments about the use of audio were received.

The following is typical of the unsolicited emails:

It is very rewarding and helpful to HEAR your comments. Now I understand more about what you are trying to say than I did with the last set of feedback we got. Thanks!

In an email received about three weeks after audio commenting was first used a student offered the following:

We've had written comments twice and verbal comments twice now. Let me guess-this is someone's research project right? Let me just save you some time. The verbal feedback is much, much, much better than the written. I said the same thing when I talked to you on campus last month. So can you just send me the voice comments from here on out, say there is no comparison between the two at all and nix the written stuff? That's probably not going to happen, but I thought it was worth a shot!

\section{B. End of Course Survey Data}

The end of course survey (response rate $=91 \%$ ) included two audio feedback specific items: one quantitative Likert-type item and one qualitative open-ended item. For the quantitative item, 26 of 31 respondents indicated that they believed audio feedback was more effective than written feedback. Four believed there was no difference between the two modalities and one responded with a N/A. The N/A response was explained in the qualitative item as described below.

When asked for additional comment related to the use of audio feedback, 11 students responded. Of these responses, 10 were highly positive and cited audio feedback as a primary reason for being satisfied with the course.

I usually find online classes rather boring. That was not the case here. It was definitely because of the way the instructor communicated with us using the audio PDF's. That approach made me interested for the first time in what was happening in an online class. I didn't feel like I was just jumping through the hoops when I got to hear the comments on my work.

No students provided negative comments related to the audio feedback. The response not categorized as positive addressed technical problems, clarifying the single N/A response to the quantitative item. 
I would definitely take an online course again, but I hope I can get this audio thing worked out if that is the way we will get comments in other courses. Even after working with tech support I never could get the files to play on my home computer. I did get them to play at work though. Because of this issue I didn't believe I could answer the question on audio commenting in the way it was intended and therefore said it was not applicable.

After the course was over, this student contacted us regarding her technical problems. It was discovered that a broken sound card in her home computer was at fault.

The survey data collected from other courses to address a potential novelty effect resulted in a $68 \%$ response rate. Of the 312 respondents, the mean number of previous courses with audio commenting was 1.31 (SD = 1.29) with 99 students having at least two previous courses utilizing this feedback modality. Responses to "I prefer audio feedback to text-based feedback" averaged 4.46 (SD = 0.78) corresponding with halfway between strongly agree and agree. In fact, only 9 students out of 312 strongly disagreed or disagreed with the statement. Directly addressing any potential novelty effect, there was no significant relation between the number of courses students had experienced with audio commenting and their relative preference for that feedback method (Spearman $r_{\mathrm{s}}=.07, n=312, n s$ ).

\section{Semi-Structured Interviews}

Students indicated that they preferred audio feedback to written feedback in 25 of the 27 interviews. One student had no preference and one preferred written feedback. From the 25 students who preferred audio feedback, four general themes emerged: 1) increased ability to understand nuances that might be lost in written communication, 2) feeling more involved in the course, 3) improved retention of content and 4) a belief that the instructor cared more about the student's learning. The mean number of themes expressed per interviewee was $2.28(\mathrm{SD}=0.79)$.

\section{Ability to Understand Nuance}

The most frequently expressed theme $(\mathrm{n}=19)$ was the ability to detect nuance and inflection in the audio commenting. In general, students believed that verbal feedback gave them increased insight into what the instructor was attempting to convey and that it produced a more comfortable, less formal learning environment.

This perspective is best illustrated by one student who said:

I have taken a couple of online classes and every time I would get these notes or critiques or comments back from the instructor and I would be wondering exactly what they were trying to say. I mean, I would understand what they were saying but not the way they were trying to say it. Sometimes you would wonder if they were agreeing with you or trying to figure out how to politely say you had it all wrong.

Now, when I first heard the audio feedback I was like wow! I get what he is saying to me. It was all in your voice and I understood when you were saying something like well this is good, but......

I understood then that you really liked what I was doing but were trying to tell me to add a little more, but in a good way. Now, in the first time we got feedback it was written and you said some things that were kind of the same but I thought you were really trying to bust me for not doing a good enough, you know, job. Then I looked at my grade and it was good so I couldn’t understand exactly what you 
were thinking.

Was my work not so good and you just gave me a decent grade? Or was it ok and I just didn't understand what [was being] said to me. When I heard you say something similar though the whole thing made sense.

One student, who had some online teaching experience, took an analytical approach to introspection as revealed by the following:

To answer what I think about this I need to tell you what I did. I've taught one online class for my department... well two if you count the one I am just finishing, so obviously I was fascinated when I got the first audio files along with my work. But I didn't want to just jump on it because it was something new. What I did was sit down and transcribe what you sent over and then I looked at it. I looked at it and listened to the files again and kept doing this for a while. What I realized was that its two completely different things.

I know you were saying the same things in your [audio files] and in what I transcribed, but the difference was you were saying them. When I looked at the transcription there was no stress placed on any of the words or sentences. Then I tried putting the stress there by adding in caps or exclamation marks and I wondered if I would have thought that you might have been yelling or something if I would have read it that way. What I figured out was that there is really no way that you could have gotten the same info across the same way.

This all made me think about the way my students have perceived me in courses when I write to them with comments. It's not the same is it? No, it's really not. We lose so much in the written word sometimes and I think maybe we haven't thought about that enough in our online teaching. [Online courses] are going to become ever more, uhm, you know, prevalent for all types of learners and I think we really need to figure out the best way to get our intent across. I think this is probably a really good first step. I know there are some things coming down the line that will make this look like we are taking baby steps, but they are steps I think we need to start taking so we can keep moving in the right direction. In a direction where we don't get dehumanized and our students don't lose what we are trying to get to them... or the way we are trying to get it to them.

\section{Feelings of Increased Involvement}

The belief that audio feedback increased feelings of being more involved and "a real part" of the class was the second most commonly expressed theme $(n=15)$. Though students often began their discussions of involvement in general terms, subsequent probing revealed that this perception was usually related to what they believed to be a lessening of social distance when audio was used.

The richest data related to this perception came from a student who cited her feeling of being more involved as the primary reason for preferring audio feedback. Her response was as follows:

Yes, I would have to say that audio [commenting] made all the difference in the world to me. I've taken several online classes here and at [another university] because they are so much more... uhm, easier for me to get to. The downside is that I have felt like I am the girl in the bubble. Some of the instructors have done these things like the biography postings and online groups that help you meet other students and get to know them; some haven't. But even where they have [used these types of activities] you still feel like you are at home in your own little bubble and you are telegraphing out to all these other bubbles that other people are sitting in. Then between all of you there is this cold wall 
type thing. It's the course, the technology, all of that stuff that makes the course. There is this barrier there.

Now, some of that has went away a little when we did things like be in chats, but it's still all kind of unreal you know? Being an Art teacher and having done my undergrad at a [very liberal college] I suppose I've always been one to seek out some of that personal interaction. So, because of that I've always felt that these online classes are a little, you know, dehumanizing.

That said, I get this file where you put in this audio and boom! It was all a big change for me you know? It was like that bubble started getting popped in all these different places and made me feel like you were reaching in there and touching me. I know that's probably kind of silly, but just your voice alone made me feel like it was a real class and not this big technology construct that was locking us into its parts.

This really changed the way I viewed the whole online learning thing. I know we aren't looking at learning the way that Judy Jetson might be learning but this tells me that we are moving that way. We are starting to reach out to each other across our phone lines and I think that's really important you know? I wish we could be doing this with each other as well as just you sending us these clip things and all. Like when we did our group projects, if we could have talked to each other like this it would have been a whole Brave New World thing going on between us but in a really good way.

Guys, keep doing this kind of stuff. Next semester and I'm done with my masters and I didn't know if I would every take another online class or not, but if I could see a class where this was going on between me and the instructor and me and the other [students] then I would be all about learning this way.

Another student who cited feelings of increased involvement was less eloquent in her initial response when she simply answered:

The audio, well, I also like it because it makes me feel like a real part of the class. You don't feel like a number when you get that.

However, subsequent probing revealed much more about her perceptions:

Here's the thing, we get all these written comments back and they are all really dense and dry. At least they seem dry. This goes back to what I meant about the inflection in the instructor's voice. When you get this written feedback it could be something where maybe the instructor has taught this course lots of times before and has all of these canned responses ready on a Word file and just cuts and pastes them into our work to save all [of their] time. I know that's probably not what's going on, at least I hope it's not, but sometimes you can feel that way. You feel you might have a robot responding to you.

What's different though with the audio though is that you know that its not canned. It could even be the same comments, but the delivery makes you feel like you are part of this learning group and that makes it all good. It makes you want to be involved, because you have this involvement level that is going to be coming back at you. 


\section{Content Retention}

For students $(\mathrm{n}=12)$ who cited increased learning and content retention as reasons for preferring audio to written feedback, most $(n=9)$ related their preference to learning style. The following is typical of students in this category:

I think the reason I like the comments made with the audio thing is that I learn better that way. Let's take when I'm in a lecture class. I look around and everyone takes all these notes but I set there and listen and record what's being said. Then when I'm studying I listen to the recording over again. I just retain better that way. With this feedback its just an extension of that; the audio I retain the first time, the written I might read four or five times.

For the remaining three students who cited increased retention with audio feedback, the following is representative:

I like this [audio feedback] because I am listening to what you are saying and scanning what I wrote. I can see what you are talking about and it clicks that way. Now, granted, I might have to listen to it and read it two or three times because doing both at once makes it all not stick as well, but in the end it works better than if both parts had been written only.

Interestingly, no students expressed a dislike for audio feedback because of learning styles. However, four did express views similar to the following:

What I find... well odd, is that I've taken learning style inventories and I know that I am very, very visual. Based on that you would think that I wouldn't like this type of feedback at all. I know that I should be liking the written comments much more, but that wasn't the case. I can read comments once and I remember. Here I was listening twice, sometimes three times to what you said to make sense of it all. However, it goes back to what we talked about earlier about feeling like I was part of the class, a real part. That offset by far the whole learning styles issue. I guess its like when we are in the classroom, we feel like the teacher is telling us something and bringing us into a discussion so we don't expect them to write it too. Maybe that's what's going on here. Maybe because you made me feel more like I was part of the class I didn't feel like I necessarily needed everything presented in the way that I learn the best.

\section{Instructor Caring}

The final theme expressed by students $(n=10)$ was related to the degree they perceived the instructor to care about their learning when audio versus written feedback was provided. In most instances $(n=8)$, this perception was closely associated with nuance and feelings of involvement as eloquently expressed by one student when she said:

The final thing is about the way I think the audio shows that you cared about us. It's not really something that's out there by itself though so I need to talk about the whole picture if that's alright with you?

I started talking about all of this by talking about feeling the tone of your voice and knowing more about what you were trying to say than when I got just the words on paper... err rather on screen... well whatever. We can start there and then when I got to understand what you were saying it gave me some idea of who you were and that made me want to be more involved. Then when I started feeling really involved and all it made me feel like you really cared about what was going on. That's a warm fuzzy I haven’t gotten with online classes before. 
A similar sentiment, though expressed quite differently, was provided by another student who said:

You took the time to try out this new audio file thing and actually communicate with us. Earlier I told you how I thought that it was way better than just reading words that might be misunderstood. That's true and so is the part when I said it made those connections that brought the class together. But what I left out is that it also showed that you were interested in our, in us learning what was going on. When you take the time to establish something that's this complex it shows you want us to really be a class and not just a group of individuals all doing something similar. I know teaching is pretty thankless, but I do want you to know that I appreciate what went on this semester. I can't really say that I've said that about any of my other online classes, but you talking to me, I mean really talking to me, and everything that was built up from that, made me feel that way here.

From the 51 questionnaires (100\% return rate) sent to students in 17 other courses where audio feedback was used in order to address any potential novelty effect, the same themes emerged with slightly different weighting than in the original study. No new themes were revealed. The prevalence of themes is presented in the following table:

\begin{tabular}{||l|c|}
\hline Theme & Prevalence \\
\hline Ability to Understand Nuance & 42 \\
\hline Feelings of Increased Involvement & 26 \\
\hline Content Retention & 27 \\
\hline Instructor Caring & 32 \\
\hline
\end{tabular}

Table 2. Prevalence of Themes in Follow-Up Questionnaires

\section{Document Analysis}

Final projects were analyzed in terms of relative usage of strategies for which audio or text feedback was provided. Two measures were used in this process to assess both frequency and level of use.

The assignment required students to use a minimum of three strategies that had been covered during the semester in completing their final project. The mean number of strategies used across five groups was 4.2 $(S D=1.09)$. The number of strategies incorporated into final projects after having received audio versus written feedback is provided in Table 3.

\begin{tabular}{||l|c|c|c||}
\hline \hline & $\begin{array}{c}\text { Total Number of } \\
\text { Strategies }\end{array}$ & $\begin{array}{c}\text { Strategies for Which } \\
\text { Audio Feedback Was } \\
\text { Received }\end{array}$ & $\begin{array}{c}\text { Strategies for Which } \\
\text { Written Feedback Was } \\
\text { Received }\end{array}$ \\
\hline Group 1 & 4 & 3 & 1 \\
\hline Group 2 & 4 & 4 & 0 \\
\hline Group 3 & 3 & 1 & 2 \\
\hline Group 4 & 4 & 3 & 1 \\
\hline Group 5 & 6 & 4 & 2 \\
\hline
\end{tabular}

Table 3. Comparison of Strategies Used in Final Projects by Feedback Type Received

Coding of documents revealed that students were far more likely to apply higher order thinking and problem solving skills (Synthesis and Evaluation in Bloom's Taxonomy) to content for which they had 
received audio feedback. Table 4 depicts the level at which strategies were applied in final projects disaggregated by the type of feedback received for those strategies.

\begin{tabular}{|l|c|c|c|c|c|c||}
\hline & Knowledge & Comprehension & Application & Analysis & Synthesis & Evaluation \\
\hline Written & 1 & 2 & 1 & 1 & 0 & 1 \\
\hline Audio & 1 & 1 & 2 & 0 & 5 & 6 \\
\hline
\end{tabular}

Table 4. Comparison of Level of Application by Feedback Type Received

\section{E. Comparison Time Requirements and Quantity of Feedback for Audio vs. Text}

During the course of the semester, 204 documents containing text-based feedback and 170 containing audio feedback were generated. The mean feedback volume for text feedback was 129.75 words (SD = 57.43) and 331.39 (SD = 89.31) for audio. The mean time required for the instructor to provide feedback, was 13.43 minutes $(\mathrm{SD}=4.53)$ for text-based feedback and 3.81 minutes $(\mathrm{SD}=0.76)$ for audio. The time required to read the documents prior to / during commenting did not differ significantly as a function of the feedback modality used. The mean time for reading the documents when text-based feedback was used was 14.13 minutes $(\mathrm{SD}=5.45)$ and 13.94 minutes $(\mathrm{SD}=5.74)$ when audio feedback was used. The average file size for audio feedback was $258 \mathrm{~kb} / \min (\mathrm{SD}=23.21)$.

\section{DISCUSSION AND CONCLUSIONS}

Our investigations revealed an overwhelming student preference for asynchronous audio feedback as compared to traditional text based feedback, with no negative perceptions of the technique. The fact that over one third of students cited the use of audio feedback as a key factor they would use in selecting future online courses is significant. When these findings are combined with data comparing the use of knowledge constructed using audio feedback and the level at which that knowledge was applied, we believe asynchronous audio commenting merits serious consideration in the development and delivery of future courses.

Though students can project themselves and their emotions through text based communication [15, 16, 20, 21], two thirds of students $(n=19)$ in this study cited ability to understand nuance as reason for preferring audio to text feedback. This finding is important because it extends upon Richardson and Swan's [22] social presence research, in which a strong relation $\left(\mathrm{R}^{2}=0.36\right)$ was found to exist between students' perceptions of social presence and satisfaction with the instructor. In addition, it is likely that an enhanced ability to detect nuance impacts student perceptions of the instructor's use of humor, and openness toward and encouragement of student ideas and discussion; key immediacy behaviors cited by Arbaugh [23].

The second most commonly expressed theme, increased feelings of involvement, is important because it reinforces the sense of community and perception of "being there." In terms of how audio commenting decreased social distance for students, the best example can be found in words offered by one student:

It was like that bubble started getting popped in all these different places and made me feel like you were reaching in there and touching me.

We consider the role audio feedback played in developing this type of interpersonal relationship with students in our asynchronous courses to be a compelling enough reason for its continued use even if no 
other positive factors had been discovered.

Findings related to perceptions of increased caring on the part of the instructor, a theme that was frequently tied to nuance and increased involvement, confirm opinions held by Olsen [31] from his use of the technique in the traditional classroom. Though students were hesitant to explore this theme in great detail during the initial interviews, it was apparent that it was of considerable importance and increased overall satisfaction with the course and the instructor. The significant increase in the percentage of students expressing this theme in follow-up questionnaires in subsequent courses is worth noting. We believe that audio feedback should be considered a means by which to increase positive perceptions of the quality of instructor interactions and, by extension, social presence in ALN.

While the preceding three themes support our contention that asynchronous audio feedback increased teaching presence and decreased social distance, it may be even more important to examine the positive impact the technique had on perceived learning. Though slightly less than half of all respondents, in both the original and follow-up interviews, indicated that they retained information and were able to synthesize instructor comments better when they received audio feedback, document analysis in the original study indicated that the impact may have been even greater.

Random assignment was used to determine whether audio or text feedback was utilized for each topic and our analysis revealed no differences in difficulty for topics assigned to each type of feedback. Even given that control, information for which audio feedback was provided was used approximately $350 \%$ more frequently than information for which text based feedback was provided. With respect to level of application, students applied content for which audio feedback was provided at the two highest levels of Bloom's Taxonomy in slightly more than $70 \%$ of the cases. In contrast, content for which text based feedback was provided was only explored at similar levels in less than $20 \%$ of cases (see Table 2). Not only did students retain material better when they received audio commenting on it, but they applied that content in more cognitively complex ways.

These findings indicate that audio feedback enhanced learning for our students; though much more research needs to be conducted to determine how generalizable these finding may be across subject matter, instructors, and institutional contexts. Since the completion of this study, other early adopters in our College have experimented with audio feedback following the techniques we employed. The quantitative, qualitative and anecdotal evidence has been overwhelmingly positive. Over 450 students in courses taught by these instructors have now received audio feedback. According to these instructors, approximately one third of their students have submitted unsolicited feedback expressing a strong preference for this technique over text based feedback. No negative feedback has been received.

From the instructors' perspective, the ability to reduce the time required to provide feedback by approximately $75 \%$ was a compelling reason to adopt the technique. However, it is important to note that this reduction in time was coupled with a $255 \%$ increase in the quantity of feedback provided. While increases in quantity of feedback delivered with less demand on instructors' time is a strong reason to use the technique, evidence that it also increased retention and understanding of content at deeper levels makes it hard to argue against using audio commenting at this point. Still, more research is needed to determine potential differences in the types of feedback provided when text-based and audio feedback are used, and the precise mechanisms that facilitate increases in student learning. 


\section{REFERENCES}

1. Berge, Z. L. New Roles for Learners and Teachers in Online Education, 2001. http://www.globaled.com/articles/BergeZane2000.pdf.

2. Liu, X., C. J. Bonk, R. J. Magiuka, S. Lee, and B. Su. Exploring four dimensions of online instructor roles: A program level case study. Journal of Asynchronous Learning Networks 9(4): 2948, 2005. http://www.sloan-c.org/publications/jaln/v9n4/v9n4 liu.asp.

3. Bennett, S. and L. Lockyer. Becoming an online teacher: Adapting to a changed environment for teaching and learning in higher education. Educational Media International 41(3): 231-244, 2004.

4. Goodyear, P., G. Salmon, J. M. Spector, C. Steeples, and S. Tickner. Competences for online teaching: A special report. Educational Technology Research \& Development 49(1): 65-72, 2001.

5. Salmon, G. E-moderating: The Key to Teaching and Learning Online. London: Taylor \& Francis, 2000.

6. Berge, Z. L. Facilitating Computer Conferencing: Recommendations from the Field. Educational Technology 15(1): 22-30, 1995.

7. Bonk, C. J., J. R. Kirkley, N. Hara, and N. Dennen. Finding the Instructor in Post-secondary Online Learning: Pedagogical, Social, Managerial, and Technological Locations. In J. Stephenson (Ed.), Teaching and Learning Online: Pedagogies for New Technologies, 76-97. London: Kogan Page, 2001.

8. Arbaugh, B. Is there an optimal design for online MBA courses? Academy of Management Learning \& Education 4(2): 135-149, 2005.

9. Dreyfus, H. On the Internet: Thinking in Action. London: Routledge, 2001.

10. Ward, M. and D. Newlands. Use of the Web in undergraduate teaching. Computers and Education 31(2): 171-184, 1998.

11. Bullen, M. Participation and critical thinking in online university distance education. Journal of Distance Education 13(2): 1-32, 1998.

12. Collis, B. Tele-Learning in a Digital World: The Future of Distance Learning. London: International Thomson Computer Press, 1996.

13. Vygotsky, L. Mind in Society: The Development of Higher Psychological Processes. Cambridge, MA: Harvard University Press, 1978.

14. Short, J., E. Williams and B. Christie. The Social Psychology of Telecommunications. London: John Wiley and Sons, 1976.

15. Rourke, L., T. Anderson, D. R. Garrison, and W. Archer. Assessing social presence in asynchronous text-based computer conferencing. Journal of Distance Education 14(2): 50, 2001.

16. Swan, K. Building communities in online course: The importance of interaction. Education, Communication and Information 2(1): 34-49, 2002.

17. Lombard, M. and T. Ditton. At the heart of it all: The concept of presence. Journal of Computer Mediated Communication 3(2): 1997. http://jcmc.indiana.edu/vol3/issue2/lombard.html.

18. Laffey, J., G. Lin and Y. Lin. Assessing social ability in online learning environments. Journal of Interactive Learning Research 17(2): 163-177, 2006.

19. Doursih, P. Where the Action Is: The Foundations of Embodied Interaction. Cambridge, MA: MIT Press, 2001.

20. Gunawardena, C. and F. Zittle. Social presence as a predictor of satisfaction within a computermediated conferencing environment. The American Journal of Distance Education 11(3): 8-26, 1997.

21. Rovai, A. A preliminary look at the structural differences of higher education classroom communities in traditional and ALN courses. Journal of Asynchronous Learning Networks 6(1): 41-56, 2002.

22. Richardson, J. and K. Swan. Examining social presence in online courses in relation to students' perceived learning and satisfaction. Journal of Asynchronous Learning Networks 6(1): 68-88, 2002.

23. Arbaugh, J. How instructor immediacy behaviors affect student satisfaction and learning in webbased courses. Business Communication Quarterly 64(4): 42-54, 2001. 
24. Gorham, J. The relationship between verbal teacher immediacy behaviors and student learning. Communication Education 37(1): 40-53, 1988.

25. Rice, R. Media appropriateness: Using social presence theory to compare traditional and new organizational media. Human Communication Research 19(4): 451-484, 1993.

26. Tang, J. and E. Isaacs. Why do users like video? Studies of multimedia-supported collaboration. Computer Supported Cooperative Work: An International Journal 1(3): 163-196, 1993.

27. Liaw, S. and H. Haung. Enhancing interactivity in Web-based instruction: A review of the literature. Educational Technology 39(1): 41-51, 2000.

28. Watt, J., J. Walther and K. Nowak. Asynchronous videoconferencing: A hybrid communication prototype. Proceedings of the 35th Hawaii International Conference on System Science, 2002.

29. Walther, J. and J. Burgoon. Relational communication in computer-mediated interaction. Human Communication Research 19(1): 50-88, 1992.

30. Jelfs, A. and D. Whitelock. The notion of presence in virtual learning environments: What makes the environment "real.” British Journal of Educational Technology 31(2): 145-152, 2000.

31. Olson, G. Beyond evaluation: The recorded response to essays. Teaching English in the Two-Year College 8(2): 121-123, 1982.

32. Mellen, C. and J. Sommers. Audio-taped responses and the two-year-campus writing classroom: The two-sided desk, the guy with the ax, and the chirping birds. Teaching English in the Two-Year College 31(1): 25-39, 2003.

33. Kim, E. The effects of digital audio on social presence, motivation and perceived learning in asynchronous learning networks. Dissertation, 2005. http://www.library.njit.edu/etd/2000s/2005/njitetd2005-075/njit-etd2005-075.html.

34. Reeves, B. and C. Nass. The Media Equation. New York: Cambridge University Press, 1996.

35. Bargeron, D., J. Grudin, A. Gupta, E. Sanocki, F. Li and S. Leetiernan. Asynchronous collaboration around multimedia applied to on-demand education. Journal of Management Information Systems 18(4): 117-145, 2002.

36. Flahery, L. and K. Pearce. Internet and face to face communication: Not functional alternatives. Communication Quarterly 46(3): 250-268, 1998.

37. Noble, D. Digital diploma mills: The automation of higher education. First Monday 3(1): 1998. http://firstmonday.org/issues/issue3_1/noble/index.html.

38. Creswell, J. W, V. L. Plano Clark, M. L. Gutmann and W. E. Hanson. Advanced mixed methods research designs. In A. Tashakkori and C. Teddlie (Eds.), Handbook of Mixed Methods in Social and Behavioral Research. Thousand Oaks, CA: Sage Publications, Inc., 2002.

39. Morse, J. M. Approaches to qualitative-quantitative methodological triangulation. Nursing Research 40: 120-123, 1991.

40. Morgan, D. L. Paradigms lost and pragmatism regained: Methodological implications of combining qualitative and quantitative methods. Journal of Mixed Methods Research 1(1): 48-76, 2007.

41. Denzin, N. K. and Y. S. Lincoln (Eds.). Collecting and Interpreting Qualitative Materials, $2^{\text {nd }}$ Edition. Thousand Oaks, CA: Sage Publications, Inc., 2003.

42. Marshall, C. and G. B. Rossman. Designing Qualitative Research. Thousand Oaks, CA: Sage Publications, Inc., 1989.

43. Searle, C. Quality in qualitative research. In Y. S. Lincoln and N.K. Denzin (Eds.), Turning Points in Qualitative Research: Tying Knots in a Handkerchief. Thousand Oaks, CA: Sage Publications, Inc., 2003.

44. Spencer, D. Student survey. 2001. http://www.sloan-c-wiki.org/wiki/index.php?title=Instructor interview_guide.

45. Thompson, M. Student post-course questionnaire. 1999. http://www.sloan-c-wiki.org /wiki/index.php?title=World_Campus_Course_Outcomes_Survey:_Spring_2000\&action=submit.

46. Gibson, C. and T. Gibson. Lessons learned from 100+ years of distance learning. Adults Learning 7(1): 15, 1995. 
47. Berg, B. L. Qualitative Research Methods for the Social Sciences. Boston, MA: Pearson Education Inc., 2004.

48. Patton, M. Q. Qualitative Evaluation and Research Methods. Thousand Oaks, CA: Sage Publications, Inc., 1990.

49. Strauss, A. L. Qualitative Analysis for Social Scientists. New York: Cambridge University Press, 1987.

50. Tesch, R. Qualitative Research: Analysis Types and Software Tools. New York: Falmer, 1990.

51. Woolfolk, C. Educational Psychology, $10^{\text {th }}$ Edition. Boston: Allyn and Bacon, 2006.

52. Slavin, R. Educational Psychology: Theory and Practice, $7^{\text {th }}$ Edition. Boston: Allyn and Bacon, 2002.

53. Ryan, G. W. and H. R. Bernard. Data management and analysis methods. In: N. K. Denzin and Y.S. Lincoln (Eds.), Collecting and Interpreting Qualitative Materials, $2^{\text {nd }}$ Edition. Thousand Oaks, CA: Sage Publications, Inc., 2003.

\section{AUTHOR BIOGRAPHIES}

Philip Ice is Clinical Assistant Professor in the College of Education's Department of Middle, Secondary and K-12 Education at the University of North Carolina Charlotte. His research interests include immediacy behaviors, pedagogy and multimedia applications in ALN. Philip is especially interested in the intersection of these elements as they relate to the Community of Inquiry model.

Reagan Curtis is an Assistant Professor of Educational Psychology in the College of Human Resources and Education's Department of Technology, Learning and Culture at West Virginia University. A research and evaluation methodologist, his research agenda is diverse including online course development and delivery, cognitive development in mathematics, and gender issues in science learning among other areas.

Perry Phillips is an Associate Professor in the College of Human Resources and Education's Department of Curriculum \& Instruction/Literacy Studies at West Virginia University. He received his doctorate in Curriculum and Instruction with a specialization in social studies education. His current research interests include teaching presence and pedagogy in ALN.

John Wells is an Associate professor of Technology Education in the School of Education at Virginia Polytechnic and State University. His line of research has been in two distinct fields: Instructional Technology Integration and Problem-Based Interdisciplinary Science and Technology Methods. John's current research interests are aimed at better understanding the intersection of learning theory and interdisciplinary STEM (science/technology/engineering/mathematics) instructional practices. Prior to Virginia Tech he was an associate professor at West Virginia University (WVU) where he served as Director of the Trek 21: Educating Teachers As Agents Of Technological Change PT3 (US Department of Education) project, the Technology Education Biotechnology Curriculum Project (NASA), and Director of the Teaching and Learning Technologies Center of the College of Human Resources \& Education at WVU. While faculty at WVU he developed and taught graduate courses related to the application of computer-mediated communication in education, web-based instructional design, transportation systems, appropriate technology, housing and shelter design, and community development. 


\section{APPENDIX A}

\section{Interview Protocol Guide}

Good morning/afternoon/evening. The goal of this study is to examine some of your observations related to the course you have just completed, C\&I 687, and the auditory feedback mechanisms that were used. The information generated by the study will be used in a research project that is designed to benefit both students and faculty with respect to the use of this medium. With your permission, I would like to audiotape this interview.

Before we begin, I would like to notify you of the following:

- Your participation is entirely voluntary. You may halt the interview at any time and/or choose not to answer certain questions.

- Your responses will remain anonymous. Complete confidentiality will be maintained. At no time will your identity be revealed either by the procedures of the study or during reporting of the results.

- No negative consequence will result for choosing not to participate.

Please feel free to tell us what you really think and feel; this will be the most helpful in trying to find out how to improve things for students and faculty members in the future.

Thank you for your participation in this research.

[Note code number and start recording.]

1. What was your overall perception of C\&I 687?

(probe for each one: 1 . likes and dislikes 2. time required to complete assignments)

2. How did the course compare with traditional courses you have taken?

(probe for: 1 . activity types 2 . interaction)

3. How did the course compare with other online courses you have taken (if any)?

(probe for differences as needed)

4. How effective, in your experience, is online learning as opposed to f2f?

(probe for: 1 . quality of discussion 2. quality of products 3. quality of interaction 4. other concerns)

5. What did you think of the types of feedback used in the course?

(probe for individual versus group responses and auditory versus written media)

6. When you think about the auditory feedback that was used, how would you describe your reaction to the instructor comments as opposed to written feedback?

(probe as needed)

7. Do you think that auditory feedback is more or less personal than written feedback? 
(probe as needed)

8. Other than what we have discussed, what did you like or dislike about auditory feedback?

(probe as needed)

9. Are there any ways in which you believe that audio feedback impacted your ability to construct knowledge in this course?

(probe as needed)

10. That is all I have. Is there anything else you would like to add?

Thank you for participating.

\section{APPENDIX B}

\section{Final Project Rubric}

Knowledge: Students explain the step-wise procedures for delivering instruction using a specific teaching strategy. Syntax is in the appropriate order; however, there is no elaboration on the methodology employed.

Comprehension: Students expand on the syntax of various teaching strategies by describing the model, as it is applied to their lesson plans, by explaining key concepts, predicting outcomes or identifying key issues that influence student learning.

Application: Students clearly apply their knowledge of teaching strategies to the content area; defined as content pedagogy.

Analysis: Students break down lesson plans into component parts and analyze the strategies employed. As an example a student would match the syntax of a given teaching strategy to the goals and objectives of activity.

Synthesis: Students apply prior knowledge from content and curriculum studies to the teaching strategy. Indicators will include inclusion of modifications to the primary teaching strategy that require the inclusion of innovative designs or combining multiple strategies into a single construct.

Evaluation: Students include, in their lesson plans, discussion elements in which judgments are made and justified by the inclusion of a set of criteria. Terminology such as compare, summarize, decide and asses are likely to be present in such discussions.

\section{APPENDIX C}

\section{Student Satisfaction Questionnaire}

The goal of this study is to examine some of your observations related to the course you have just completed and the auditory feedback mechanisms that were used. The information generated by the study 
will be used in a research project that is designed to benefit both students and faculty with respect to the use of this medium.

Before you complete the survey please be aware of the following:

- Your participation is entirely voluntary. You may choose to answer or not answer any or all questions.

- Your responses will remain anonymous. Complete confidentiality will be maintained. At no time will your identity be revealed either by the procedures of the study or during reporting of the results.

- No negative consequence will result for choosing not to participate.

Please feel free to tell us what you really think and feel; this will be the most helpful in trying to find out how to improve things for students and faculty members in the future.

Thank you, in advance, for participating in this study.

1. What was your overall perception of (course name and number here)? Please describe what you liked and disliked about the course.

2. How did the course compare with traditional courses you have taken? When answering this question think about the types of activities, interaction with the instructor and interaction with fellow students.

3. How did the course compare with other online courses you have taken (if any)? Please elaborate a little on differences (either positive or negative).

4. How effective, in your experience, is online learning as opposed to f2f? If, in your opinion, the following are applicable, please elaborate: 1. Quality of discussion. 2. Quality of learning. 3. Quality of interaction. 4. Any other issues you care to discuss.

5. What did you think of the types of feedback used in the course?

6. When you think about the auditory feedback that was used, how would you describe your reaction to the instructor comments as opposed to written feedback you may have received in this course or previous courses?

7. Do you think that auditory feedback is more or less personal than written feedback? Why?

8. Other than what we have discussed, what did you like or dislike about auditory feedback?

9. Do you have any other comments about the course or the instructor?

When you have completed the survey please save it as a Word document and email it to (insert email drop here). Thank you once again, for agreeing to complete this survey. 